\title{
Mavoglurant (AFQ056) for the treatment of levodopa-induced dyskinesia in patients with Parkinson's disease: a meta-analysis
}

\author{
Ahmed Negida ${ }^{1,2,3}$ (D) Hazem S. Ghaith ${ }^{1,4}$. Salma Yousry Fala ${ }^{1,5}$. Hussien Ahmed ${ }^{1,2} \cdot$ Eshak I Bahbah $^{1,6}$. \\ Mahmoud Ahmed Ebada ${ }^{1,2} \cdot$ Mohamed Abd Elalem Aziz ${ }^{1,7}$
}

Received: 31 March 2021 / Accepted: 9 May 2021 / Published online: 20 May 2021

(C) The Author(s) 2021

\begin{abstract}
Background Mavoglurant (AFQ056), a selective metabotropic glutamate receptor 5 (mGluR5) inhibitor, was tested for $\mathrm{t}$ levodopa-induced dyskinesia (LID) in patients with Parkinson's Disease (PD). However, clinical trials showed inconsistent results regarding the efficacy of mavoglurant in treating LID in patients with Parkinson's disease (PD).

Methods A computer literature search of PubMed, Scopus, Web of science, and Cochrane CENTRAL was conducted until March 2021. We selected relevant randomized controlled trials comparing mavoglurant to placebo. Study data were extracted and pooled as mean difference (MD) in the meta-analysis model.

Results Six RCTs were included in this meta-analysis with a total of 485 patients. Mavoglurant was not significantly superior to placebo in terms of the "off-time" (MD $-0.27 \mathrm{~h}, 95 \% \mathrm{CI}-0.65$ to 0.11), "on time" (MD $0.29 \mathrm{~h}, 95 \% \mathrm{CI}-0.09$ to 0.66 ), LangFahn activities of daily living dyskinesia scale (MD $-0.95,95 \%$ CI -1.98 to 0.07 ), UPDRS-III (MD $-0.51,95 \%$ CI -1.66 to 0.65 ), or UPDRS-IV (MD $-0.41,95 \%$ CI -0.85 to 0.03 ). However, the pooled modified abnormal involuntary movement scale favored the mavoglurant group than the placebo group (MD $-2.53,95 \% \mathrm{CI}-4.23$ to -0.82 ).

Conclusions This meta-analysis provides level one evidence that mavoglurant is not effective in treating the LID in patients with PD.
\end{abstract}

Keywords Parkinson's disease $\cdot$ Mavoglurant $\cdot$ Levodopa

\section{Introduction}

Parkinson's disease is a common neurological disorder affecting about $1-3 \%$ above 60 years population [1]. The loss of dopaminergic neurons and the formation of Lewy bodies are

Ahmed Negida

ahmed01251@medicine.zu.edu.eg; ahmed.said.negida@gmail.com

Medical Research Group of Egypt, Cairo, Egypt

2 Faculty of Medicine, School of Medicine, Zagazig University, Sharkia, Zagazig 44523, Egypt

3 School of Pharmacy and Biomedical Sciences, University of Portsmouth, Portsmouth, UK

4 Faculty of Medicine, Al-Azhar University, Cairo, Egypt

5 Faculty of Medicine, Suez Canal University, Ismailia, Egypt

6 Faculty of Medicine, Al-Azhar University of Damietta, Damietta, Egypt

7 Neuropsychiatry Department, Omr Shahin Mental Hospital, Cairo, Egypt the cardinal pathological features of PD. PD symptoms include rigidity, tremors, and dyskinesia due to decreased dopamine levels in the basal ganglia of PD patients. Levodopa, a precursor of dopamine, is considered as the standard of care for patients with early PD. However, PD patients do not respond optimally to levodopa. The wearing off of the drug and motor fluctuations influence the quality of life of PD patients.

At 5 years after initiation of levodopa treatment, about $50 \%$ of PD patients suffer from levodopa-induced dyskinesia (LID), requiring dose adjustments to balance the motor benefit against the related complications [2, 3]. Amantadine, a NMDA blocker, is an effective drug to treat LID; however, its short-term efficacy warranted the development of alternative add-on agents with long-term durability $[4,5]$. There is an unmet clinical need for adjuvant treatments to treat LID and allow reductions in levodopa dose with long term durability and without influencing the motor benefit of the levodopa treatment.

The mechanism of LID is not yet elucidated; however, the literature suggests that the glutamatergic pathway has a role in the emergence of LID [2, 6]. Increased glutamatergic signaling was found in the striatum of basal ganglia [7]. 
Additionally, the modulation of glutamatergic signaling by mGluR5 antagonists was found to be effective in reducing the LID in animal models [8]. Mavoglurant (AFQ056), a selective mGluR5 inhibitor, was developed to treat LID by decreasing the glutamatergic signaling in the striatum [9].

Multiple clinical trials were conducted to assess the safety and efficacy of mavoglurant for the treatment of LID in PD patients. Berg et al. [10] found a significant improvement in the Modified abnormal involuntary movement scale (mAIMS) and unified Parkinson's disease rating scale (UPDRS)-IV scores in the mavoglurant group compared to the placebo group. However, a recent study by Trenkwalder et al. [11] showed no evidence of benefit from mavoglurant.

There is a lack of class-one evidence about the safety and efficacy of mavoglurant for the treatment of LID in patients with PD. Hereby, we evaluated the safety and efficacy of mavoglurant compared to placebo for the treatment of LID in PD patients using data from randomized controlled trials pooled in the context of meta-analysis.

\section{Methods}

We followed the Preferred Reporting Items of Systematic Reviews and Meta-analysis during the preparation of this manuscript [12]. The methods and analyses were conducted in strict accordance with the guidelines of the Cochrane Handbook of Systematic Review and Meta-analysis and the Methods Guide for Comparative Effectiveness Reviews [13, 14].

\section{Eligibility criteria}

Studies that fit all of the following criteria were included in the meta-analysis:

(1) Population: Studies whose population was patients with idiopathic PD (diagnosed using the UK PD society Brain Bank Criteria) [12] and suffering from moderate to severe LID (defined as those whose Unified Parkinson's Disease Rating Scale-IV item $32>1$ and item $33>2$ ).

(2) Intervention: Studies where patients receive AFQ056 as experimental drug (all doses are considered).

(3) Comparator: Studies where the control group received placebo

(4) Study design: Studies that were described as prospective randomized controlled trials.

We excluded studies on drugs other than AFQ056.

\section{Literature search}

We searched electronic databases: PubMed, Scopus, Web of science, and Cochrane CENTRAL through March 2021, using the following keywords: "(AFQ056 OR mavoglurant OR mGluR5) AND Parkinson's disease”. No limitations or filters were employed.

\section{Study selection}

Results of databases search were screened by titles and abstracts; then full-text articles of eligible abstracts were retrieved for further eligibility screening and assessment of the reliability of data for analysis.

\section{Data collection process and data items}

An online data extraction sheet was constructed. The data extraction includes the following domains: (1) study ID, (2) study year, (3) country, (4) study design, (5) follow up duration, (6) drug dose, (7) population definition, (8) inclusion and exclusion criteria, (9) sample size, (10) baseline characteristics, (11) available data of outcome measures (pre, post, and change from baseline), and (12) quality assessment domains.

\section{Risk of bias in individual studies}

The Cochrane risk of bias assessment tool [13] is a recommended checklist for assessing the risk of bias in RCTs. In this tool, each RCT is assessed for the possibility of the following risks: (1) selection bias, by assessing the methods of random sequence generation and the concealment of patient allocation; (2) performance bias, by assessing the blinding of participants and study personnel; (3) detection bias, by assessing the blinding methods of outcome assessment process; (4) attrition bias, by evaluating the magnitude and impact of incomplete outcome data and whether incomplete data were handled by appropriate statistical analysis techniques; (5) reporting bias, by assessing the selectivity of reporting study outcomes based on the prespecified methods in the clinical trial registration; and (6) any other source of bias that might have influenced the study data.

\section{Efficacy measures}

The efficacy of drugs treating LID is assessed for the following outcomes.

\section{Patient-reported home diaries which give the following outcomes}

Patient-reported diaries include information about the duration of "off-time", "on time", "on time without dyskinesia", "on time with nontroublesome dyskinesia", and "on time with troublesome dyskinesia". 
Modified abnormal involuntary movement scale (mAIMS)

MAIMS [14] is a composite score of dyskinesia rating in seven body parts.

\section{UPDRS part 3 and part 4 (items 32 and 33)}

The unified Parkinson's disease rating scale [15] is a reliable score of four parts to assess the severity of PD symptoms. Part 3 indicates the motor score, whereas part 4 indicates the severity of $\mathrm{PD}$ complications, with items 32 and 33 referring to the duration and severity of dyskinesia, respectively.

\section{Lang-Fahn activities of daily living dyskinesia scale (LFADLDS)}

Lang-Fahn activities of daily living dyskinesia scale focus on patient perceptions of disability related to dyskinesia. An ordinal scale $(0,0$ representing no dyskinesia and 4 representing inability to perform the task) assesses. The patient's report on five activities of daily living potentially impacted by dyskinesia at their maximum severity over the past few days (handwriting or drawing, cutting food and handling utensils, dressing, hygiene, and walking).

\section{Synthesis of results}

Because all efficacy outcomes are reported as continuous data, for each efficacy measure, the mean difference (MD) between the two groups from the baseline to endpoint, with its standard error (SE) were pooled in the DerSimonian-Laird random effect model. In case of studies reporting data in multiple time points, we considered the last endpoint. The overall MD was interpreted with the consideration that efficacy measures are in different directions; the improvement in mAIMS, UPDRS IV, UPDRS III, "off time", and "on time with troublesome dyskinesia" is indicated by decreased MD while the improvement in "on time without dyskinesia" and "on time with nontroublesome dyskinesia" is indicated by increased MD. For the safety analysis, we analyzed adverse events reported with an incidence $\geq 5 \%$ in the included studies. The proportion of adverse events to the total number of patients in each group were pooled as relative risk between the two groups in the DerSimonian-Laird random effect model. Heterogeneity (noncombinability) of studies and subgroups was examined by visual inspection of the forest plot and assessed by the Cochrane $\mathrm{Q}$ and $\mathrm{I}^{2}$ tests using RevMan version 5.3 for windows.

\section{Calculation of missing data}

When the mean difference from baseline to endpoint was not provided, we calculated it from the pretreatment and posttreatment means $[\mathrm{MD}=$ Posttreatment - pretreatment $]$. Then, we calculated mean difference between the AFQ056 and placebo groups as follows: [MD $=$ MDexperiemental - MDplacebo $]$. When the SE of mean difference was not provided, we calculated it from the standard deviation $[\mathrm{SE}=\mathrm{SD} / \sqrt{ } n], 95 \%$ confidence interval [(upperlimit-lowerlimit)/3.92], or $90 \%$ CI [(upperlimit-lowerlimit)/3.29]. For studies and groups with sample size less than 60 patients, the numbers (3.92 and 3.29) were substituted by a value from the table of $t$ distributions with degrees of freedom equal to the group sample size minus 1 .

\section{Risk of bias across studies}

In order to explore the publication bias across studies, we constructed funnel plots to present the relationship between effect size and precision. Evidence of publication bias was assessed by the following: (1) Egger's regression test and (2) the Begg and Mazumdar rank correlation test (Kendall's tau).

\section{Results}

\section{Study selection}

The literature search of PubMed, Scopus, Web of science, and Cochrane CENTRAL yielded 15 records. Following titles and abstract screening, four articles describing six randomized controlled trials with a total of 485 patients were included in the meta-analysis $[10,11,15,16]$. The flow of the study selection process is shown in the PRISMA flow diagram in Supplementary File 1.

\section{Study characteristics}

The duration of follow-up in the studies ranged from 2 weeks in the Berg et al (2010) [7](study 1 and study 2) to 13 weeks in the study by Stocchi et al(2013)[17]. Patients in studies by Berg et al (2010) [7]and Kumar et al (2016) [16]received daily doses of mavoglurant ranging from 20 to $300 \mathrm{mg}$, while patients in the study by Stocchi et al (2013) were classified into five subgroups receiving mavoglurant doses of 25,50 , 100,150 , or $200 \mathrm{mg}$. In all studies, PD patients remained on the levodopa treatment during the study period.

The population of these studies was homogenous. All studies enrolled patients with Parkinson's disease (diagnosed according to UK PD society brain bank criteria) with moderate to severe LID as indicated by the UPDRS-IV (items 32 and 33). All studies excluded patients with (1) history of psychological problems, (2) prior surgical treatment for PD, and (3) use of antipsychotic or antidyskinetic drugs 15 days prior to randomization. Summary and baseline characteristics of populations of these studies are shown in Table 1 . The quality of included studies was acceptable according to the Cochrane Risk of Bias assessment tool. 
Table 1 Baseline characteristics of the study population in the included studies

\begin{tabular}{|c|c|c|c|c|c|c|c|c|c|}
\hline Study ID & Design & Final endpoint & Group & $\mathrm{N}$ & Age* & Male & mAIMS* & UPDRS-III* & $\begin{array}{l}\text { UPDRS-IV } \\
\text { (items } 32 \text { and 33)* }\end{array}$ \\
\hline \multirow{2}{*}{$\begin{array}{l}\text { Berg } 2010 \\
\text { (Study 1) }\end{array}$} & \multirow[t]{2}{*}{ RCT } & \multirow[t]{2}{*}{2 weeks } & Mavoglurant & 15 & $60.7(10.5)$ & $60 \%$ & $11.4(4.79)$ & $26.6(14.78)$ & $4.7(1.03)$ \\
\hline & & & Placebo & 16 & $61.4(10.2)$ & $43.8 \%$ & $9.1(3.87)$ & $22.1(9.77)$ & $4.1(0.89)$ \\
\hline \multirow{2}{*}{$\begin{array}{l}\text { Berg } 2010 \\
\text { (Study 2) }\end{array}$} & \multirow[t]{2}{*}{$\mathrm{RCT}$} & \multirow[t]{2}{*}{3 weeks } & Mavoglurant & 14 & $65.6(7.5)$ & $64.3 \%$ & $16(4.07)$ & $26.1(16.67)$ & $5.4(0.95)$ \\
\hline & & & Placebo & 14 & $66.1(6.5)$ & $57.1 \%$ & $16.3(4.32)$ & $26(9.68)$ & $4.8(0.89)$ \\
\hline \multirow[t]{6}{*}{ Stocchi 2013} & \multirow[t]{6}{*}{$\mathrm{RCT}$} & \multirow[t]{6}{*}{13 weeks } & Mavoglurant $20 \mathrm{mg}$ & 22 & $66.2(8.16)$ & $45.5 \%$ & $14.5(4.64)$ & $16.6(8.13)$ & $5.1(0.89)$ \\
\hline & & & Mavoglurant $50 \mathrm{mg}$ & 22 & $66.4(7.96)$ & $59.1 \%$ & $12.9(5.03)$ & $18.6(7.97)$ & $4.9(0.01)$ \\
\hline & & & Mavoglurant $100 \mathrm{mg}$ & 23 & $65.6(9.47)$ & $60.9 \%$ & $13.3(5.25)$ & $18.5(9.65)$ & $4.8(0.8)$ \\
\hline & & & Mavoglurant $150 \mathrm{mg}$ & 22 & $66(10.54)$ & $63.6 \%$ & $13.9(5.08)$ & $18.9(9.25)$ & $4.8(0.73)$ \\
\hline & & & Mavoglurant $200 \mathrm{mg}$ & 44 & $63.4(8.98)$ & $54.5 \%$ & $13.4(4.91)$ & $17.5(9.31)$ & $5(0.88)$ \\
\hline & & & Placebo & 64 & $64.8(8.17)$ & $46.9 \%$ & $13.5(4.68)$ & $16.9(9.39)$ & $4.9(0.82)$ \\
\hline \multirow[t]{2}{*}{ Kumar 2016} & \multirow[t]{2}{*}{ RCT } & \multirow[t]{2}{*}{5 weeks } & Mavoglurant & 7 & $61.3(8.98)$ & $57.1 \%$ & NR & $22.3(15.32)$ & NR \\
\hline & & & Placebo & 7 & $61.4(6)$ & $71.4 \%$ & NR & $16.1(7.8)$ & NR \\
\hline \multirow{2}{*}{$\begin{array}{l}\text { Trenkwalder } 2016 \\
\quad \text { (study 1) }\end{array}$} & \multirow[t]{2}{*}{$\mathrm{RCT}$} & \multirow[t]{2}{*}{12 weeks } & Mavoglurant $100 \mathrm{mg}$ & 36 & $65.9(6.97)$ & $52.8 \%$ & $12.5(4.88)$ & $20.5(10.13)$ & $4.8(0.87)$ \\
\hline & & & Placebo & 25 & $66.6(7.04)$ & $60.0 \%$ & $12.8(5.08)$ & $19.5(7.91)$ & $4.8(0.66)$ \\
\hline \multirow{3}{*}{$\begin{array}{l}\text { Trenkwalder } 2016 \\
\quad \text { (study 2) }\end{array}$} & \multirow[t]{3}{*}{$\mathrm{RCT}$} & \multirow[t]{3}{*}{12 weeks } & Mavoglurant $150 \mathrm{mg}$ & 39 & $64.4(8.68)$ & $56.4 \%$ & $12.38(4.178)$ & $18.05(9.409)$ & $4.67(0.898)$ \\
\hline & & & Mavoglurant $200 \mathrm{mg}$ & 78 & $64.4(8.84)$ & $52.6 \%$ & $12.59(5.062)$ & $20.26(11.319)$ & $4.82(0.833)$ \\
\hline & & & Placebo & 37 & $64.2(9.02)$ & $56.8 \%$ & $11.73(5.064)$ & $19.32(9.548)$ & $4.81(0.811)$ \\
\hline
\end{tabular}

*Continuous outcomes presented as mean (SD). mAIMS, modified abnormal involuntary movement scale; UPDRS, unified Parkinson's disease rating scale; $R C T$, randomized controlled trials; $N R$, not reported

\section{Drug efficacy}

\section{Off-time}

The overall mean difference between the two groups from baseline to endpoint in terms of change in "Off time" did not favor either of the two groups (MD $-0.27 \mathrm{~h}, 95 \% \mathrm{CI}[-0.65$ to 0.11 ], Fig. 1A). Pooled studies were homogenous $(P=0.83)$.

\section{Total on time}

The pooled mean difference of change in the total "ontime" did not favor either of the two groups (MD $0.29 \mathrm{~h}$, $95 \%$ CI $[-0.09$ to 0.66$]$, Fig. 1B). Pooled studies were homogenous $(P=0.68)$.

\section{On time without dyskinesia}

The study by Kumar et al.(2016) [15]was the only on that reported the "on time without dyskinesia". In this study, patients in the mavoglurant group had more improvement in the "on-time without dyskinesia" compared with the placebo group (mean change from baseline to endpoint in both groups: 3.83 vs. 1.42 h, respectively).

\section{On time with troublesome dyskinesia}

None of the included studies reported a statistically significant improvement in the "on time with troublesome dyskinesia" with the mavoglurant compared to placebo. The pooled mean difference of change in the "on time with troublesome dyskinesia" did not favor either of the two groups (MD $0.00 \mathrm{~h}, 95 \%$ CI [ -0.42 to 0.43$]$, Fig. 1C). Pooled studies were homogenous $(P=0.98)$.

\section{mAIMS}

In the mavoglurant group of studies 1 and 2 by Berg et al (2010) [7], and Stocchi et al (2013) [17] (group of $200 \mathrm{mg}$ ), the improvement in mAIMS score was significantly higher than that in the placebo groups. For the subgroups in the other studies, the improvement in mAIMS within the mavoglurant group was higher but not statistically significant. When combined in the metaanalysis model, the pooled mean difference of change in the mAIMS favored the mavoglurant group over the placebo group (MD $-2.53,95 \%$ CI [-4.23 to -0.82$]$, Fig. 2A). Pooled studies were not homogenous $(P=0.01$, I-square $=65 \%)$. Therefore, we performed a sensitivity analysis in seven scenarios excluding one study/subgroup in each scenario. Heterogeneity was best 


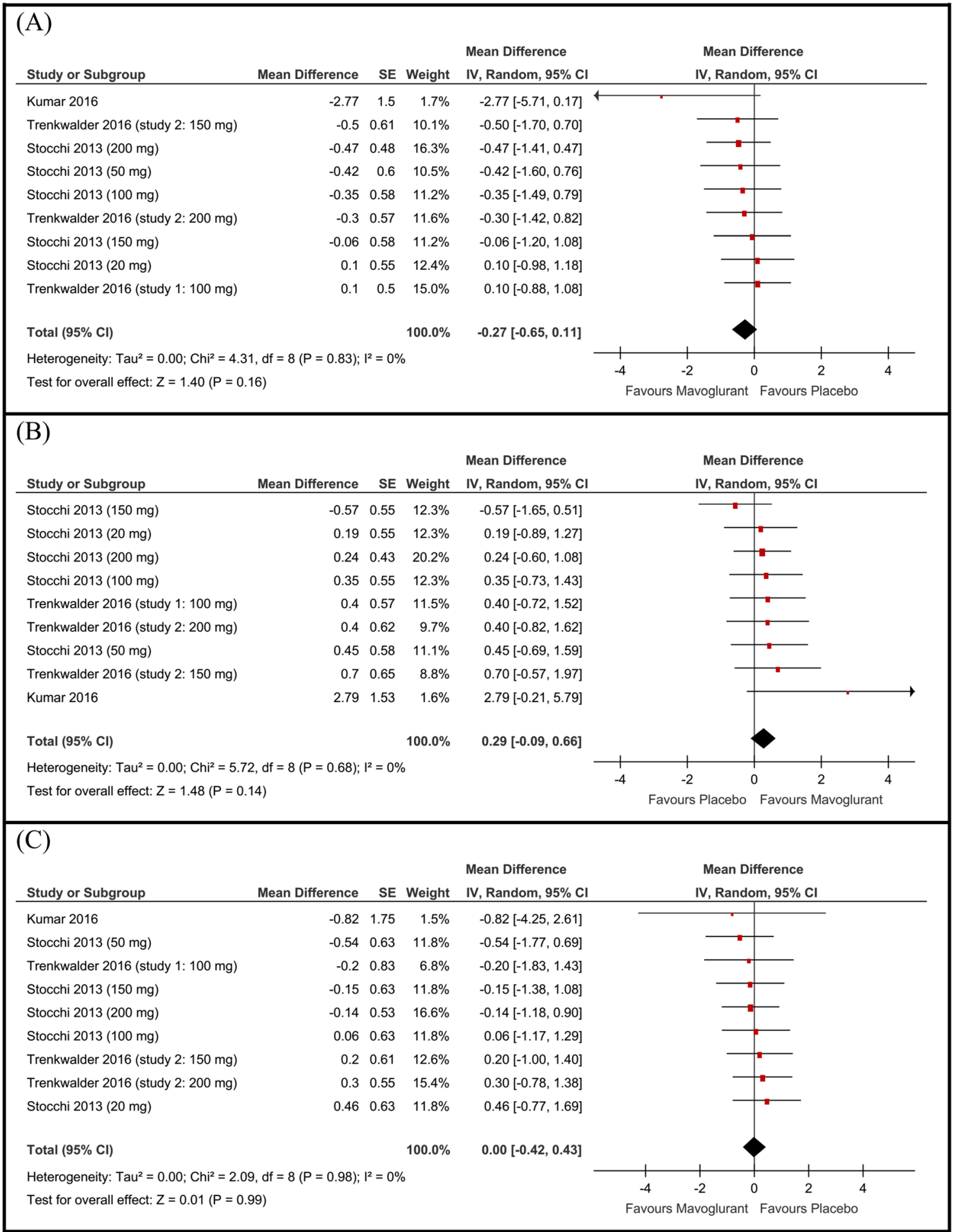

Fig. 1 Forest plot of the mean difference and 95\% confidence intervals of the $\mathbf{A}$ off time, $\mathbf{B}$ total on time, and $\mathbf{C}$ on time with troublesome dyskinesia

resolved by excluding study 1 by Berg et al (2010) [7] $\left(P=0.26, \mathrm{I}^{2}=24 \%\right)$. Following resolving heterogeneity, the effect estimate remained in favor of the mavoglurant group over the placebo group (MD -1.70 point, $95 \% \mathrm{CI}$ $[-2.98$ to -0.42$]$, Fig. 2B).

\section{Lang-Fahn activities of daily living dyskinesia scale (LFADLDS)}

The overall mean difference of change in LFADLDS from baseline to end point did not favor either of the two 


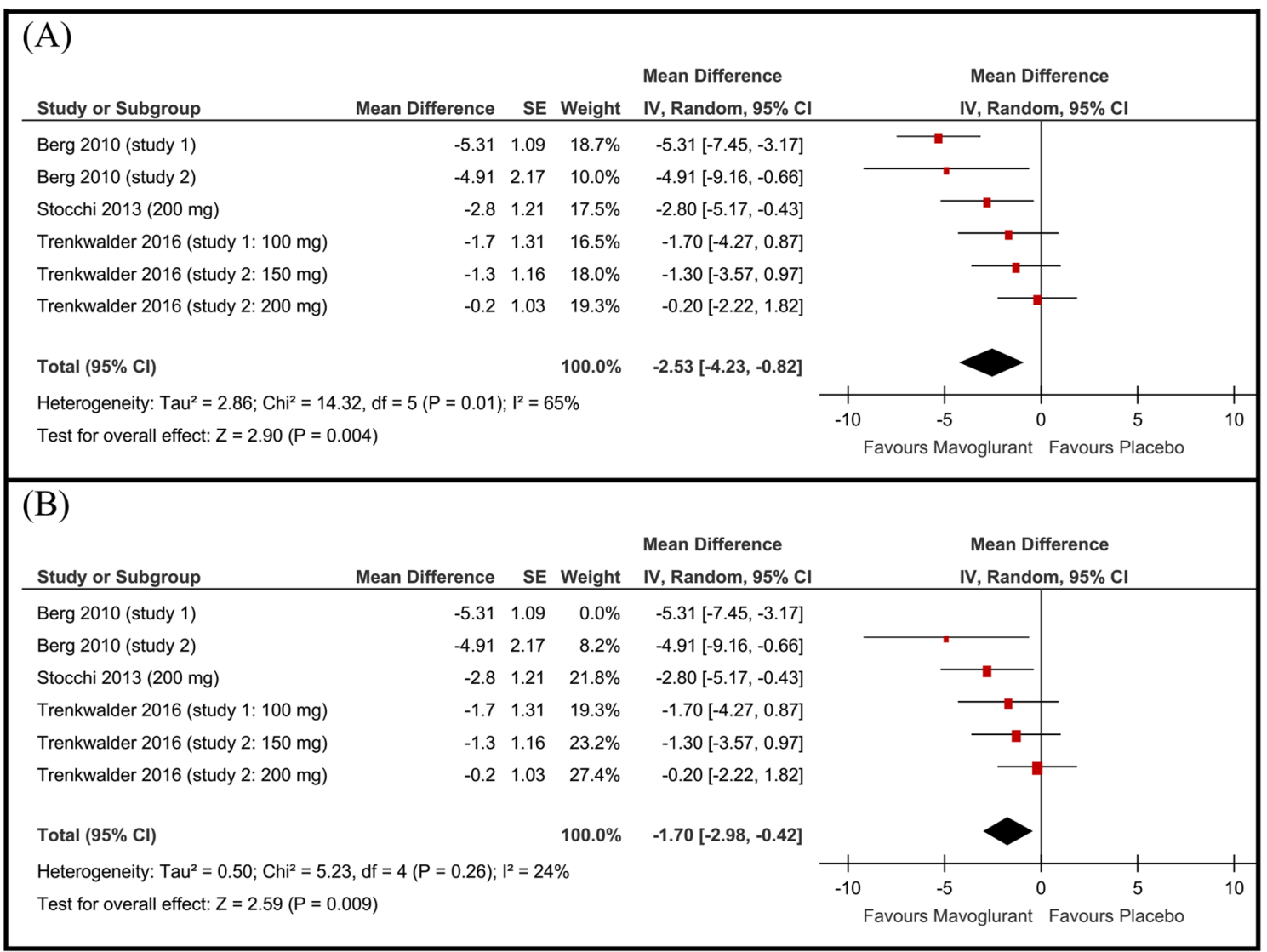

Fig. 2 Forest plot of the mean difference and 95\% confidence intervals of the A modified abnormal involuntary movement scale (mAIMS) score and B mAIMS score after sensitivity analysis excluding Berg study 1

groups (MD $-0.95,95 \%$ CI [ -1.98 to 0.07$]$, Fig. 3A), a $P$ value of 0.07 illustrated a trend toward favoring the mavoglurant group. Pooled studies were homogenous $(P=0.35)$.

\section{UPDRS-IV (items 32 and 33)}

Compared to the placebo group, the mavoglurant group achieved better scores on the UPDRS-IV (items 32 and 33). This difference was statistically significant in the Berg et al. (2010) [7] (study 1 and study 2). However, the pooled mean difference of change in the UPDRS-IV (items 32 and 33) did not favor either of the two groups (MD -0.41 point, $95 \%$ CI [ -0.85 to 0.03$]$, Fig. 3B).

\section{UPDRS-III}

All studies showed no statistically significant difference between the mavoglurant and placebo groups in terms of the change in UPDRS-III. The pooled mean difference of change in the UPDRS-III did not favor either of the two groups (MD
-0.51 point, $95 \%$ CI [ -1.66 to 0.65$]$, Fig. 3C). Pooled studies were homogenous $(P=0.97)$.

\section{Adverse events}

Of the seven analyzed adverse events, only dizziness showed a statistically significant risk in the mavoglurant group compared to the placebo group (RR 4.19, 95\% CI [1.83 to 9.64]). In all studies, patients in the mavoglurant group experienced more hallucinations, euphoria, and nausea than patients in the placebo group, however, the pooled RR was not statistically significant. Effect estimates of the adverse events with their 95\% confidence intervals are shown in Table 2.

\section{Risk of bias across studies}

Our meta-analysis included fewer than 10 studies. Therefore, the assessment of publication bias using the funnel plot method and Egger's test will not be reliable as stated by Egger's and colleagues. 


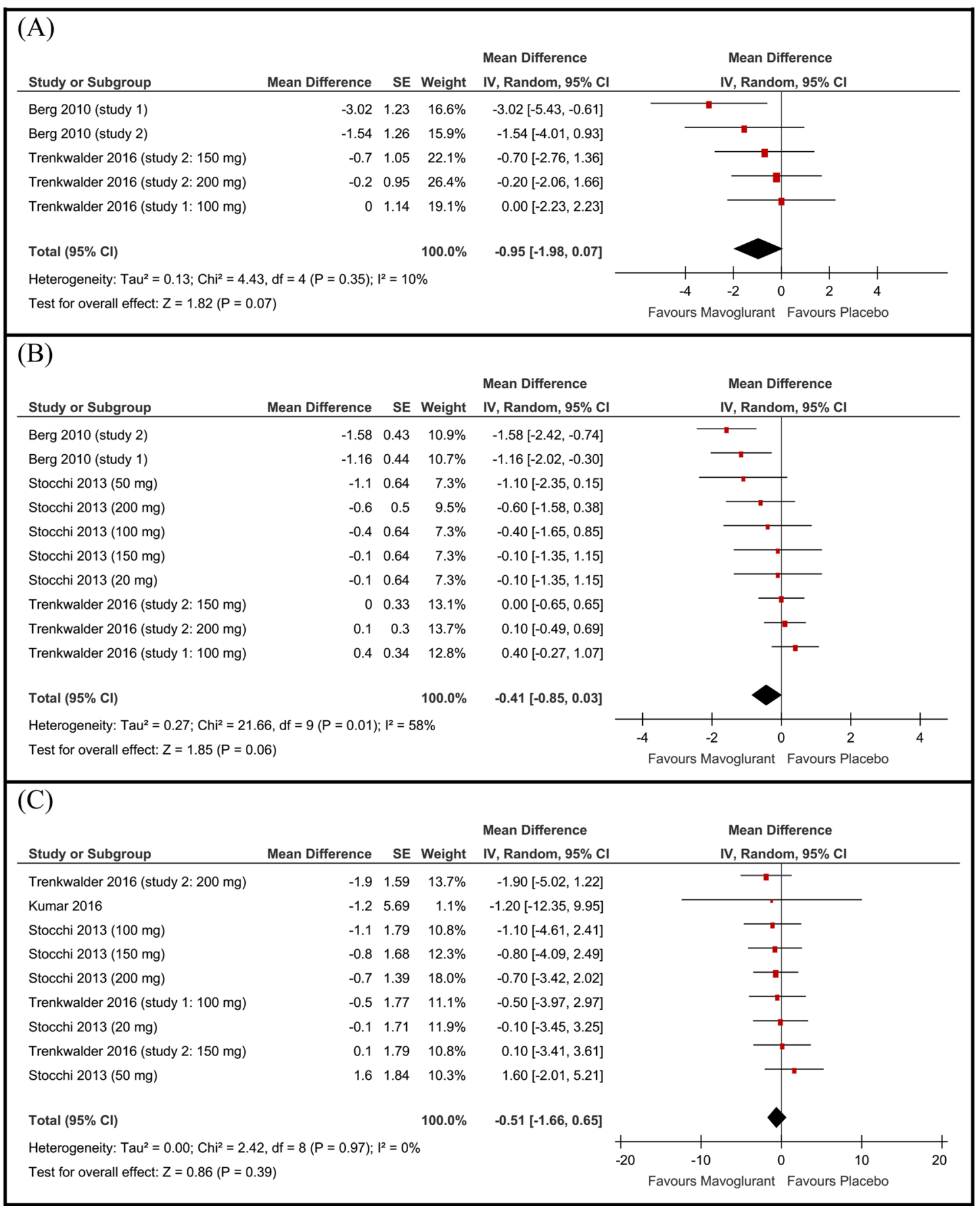

Fig. 3 Forest plot of the mean difference and 95\% confidence intervals of the A Lang-Fahn activities of daily living dyskinesia scale, B UPDRS-IV items 32 and 33, and C UPDRS-III motor score

\section{Discussion}

\section{Summary of evidence}

The present meta-analysis provides class one evidence that mavoglurant as an add-on treatment does not improve LID as reported by patient diaries and UPDRS-IV (items 32 and
33). However, it was associated with a significant improvement in the dyskinesia scores as measured by the mAIMS score. Additionally, mavoglurant did not influence the antiparkinsonian effects of levodopa as measured by the UPDRS-III. In terms of safety, the drug was associated with a fourfold increase in the incidence of dizziness compared to the placebo group. 
Table 2 Summary of the pooled risk ratios (RR) between the mavoglurant group and placebo group in all reported adverse events

\begin{tabular}{llll}
\hline Adverse event & N of RCTs & N patients & RR [95\% CI] \\
\hline Dizziness & 5 & 471 & $4.19[1.83$ to 9.64$]$ \\
Dyskinesia & 6 & 585 & $0.74[0.46$ to 1.17$]$ \\
Euphoria & 3 & 73 & $3.70[0.64$ to 21.29$]$ \\
Fatigue & 5 & 471 & $2.11[0.72$ to 6.15$]$ \\
Insomnia & 4 & 426 & $1.90[0.58$ to 6.23$]$ \\
Hallucination & 2 & 211 & $4.55[0.86$ to 24.04$]$ \\
Nausea & 4 & 274 & $2.02[0.48$ to 8.46$]$ \\
\hline
\end{tabular}

$R C T$, randomized controlled trial

\section{Previous studies}

The effect estimates produced in our meta-analysis are consistent with the previous randomized controlled trials in terms of LID measured by patient diaries. However, in terms of mAIMS and UPDRS-IV (items 32 and 33), results of randomized controlled trials were controversial. Berg et al.(2010) [7] described two RCTs (studies 1 and 2) on PD patients with moderate to severe and severe LID, respectively. Unlike other studies, patients in the studies by Berg et al.(2010) [10] showed statistically significant improvements in mAIMS and UPDRS-IV (items 32 and 33). This could be explained by the patients in the studies by Berg et al.(2010) [10] having poorer baseline UPDRS-III scores than those in other studies (baseline characteristics are shown in Table 1). This observation highlights that mavoglurant might be effective for patients with UPDRS-III $>22$. In the study by Stocchi et al.(2013) [16], patients receiving $200 \mathrm{mg}$ mavoglurant showed statistically significant improvements in mAIMS scores compared to those receiving placebo; however, this improvement was not significant for the other doses $(20,50$, 100 , and $150 \mathrm{mg}$ ). The pooled analysis showed a small but statistically significant improvement in mAIMS score, but no significant improvement was found in the UPDRS-IV (items 32 and 33).

\section{Strengths of the study}

Our meta-analysis has multiple strengths: (1) the literature search strategy was rigorous; (2) the research question was supported by clear eligibility criteria; (3) each step in the review was done by multiple reviewers to ensure accuracy; (4) we followed the Preferred Reporting Items of Systematic Review and Meta-analysis during the preparation of this manuscript; (5) we conducted this meta-analysis in strict accordance with the guidelines of Cochrane Handbook of systematic reviews and meta-analysis; and (6) the present data were generated from randomized controlled trials with high internal validity and acceptable quality as indicated by the risk of bias assessment.

\section{Limitations of the study}

The limitations of this meta-analysis are the following: (1) the limited number of studies did not allow for investigation of the effect of dose on patient outcomes and (2) the limited sample size in the present RCTs.

\section{Implications for future research}

Based on this meta-analysis, we recommend no future randomized controlled to investigate the efficacy mavoglurant for PD patients suffering from LID. We do not recommend the use of this drug in the clinical practice.

\section{Conclusions}

Current evidence does not support the efficacy of mavoglurant for the treatment of LID in PD patients. We do not recommend the use of this drug for LID in PD patients.

Supplementary Information The online version contains supplementary material available at https://doi.org/10.1007/s10072-021-05319-7.

Data availability All data underlying the results are available as part of the article, and no additional source data are required.

\section{Declarations}

Ethical approval None.

Conflict of interest The authors declare that they have no competing interests.

Open Access This article is licensed under a Creative Commons Attribution 4.0 International License, which permits use, sharing, adaptation, distribution and reproduction in any medium or format, as long as you give appropriate credit to the original author(s) and the source, provide a link to the Creative Commons licence, and indicate if changes were made. The images or other third party material in this article are included in the article's Creative Commons licence, unless indicated otherwise in a credit line to the material. If material is not included in the article's Creative Commons licence and your intended use is not permitted by statutory regulation or exceeds the permitted use, you will need to obtain permission directly from the copyright holder. To view a copy of this licence, visit http://creativecommons.org/licenses/by/4.0/.

\section{References}

1. Pringsheim T, Jette N, Frolkis A, Steeves TDL (2014) The prevalence of Parkinson's disease: a systematic review and meta-analysis. Mov Disord 29:1583-1590. https://doi.org/10.1002/mds.25945 
2. Jankovic J (2005) Motor fluctuations and dyskinesias in Parkinson's disease: clinical manifestations. Mov Disord 20:S11S16. https://doi.org/10.1002/mds.20458

3. Arabia G, Zappia M, Bosco D, Crescibene L, Bagalà A, Bastone L, Caracciolo M, Scornaienghi M, Quattrone A (2002) Body weight, levodopa pharmacokinetics and dyskinesia in Parkinson's disease. Neurol Sci 23:s53-s54. https://doi.org/10.1007/s100720200066

4. da Silva-Júnior FP, Braga-Neto P, Sueli Monte F, de Bruin VMS (2005) Amantadine reduces the duration of levodopa-induced dyskinesia: a randomized, double-blind, placebo-controlled study. Parkinsonism Relat Disord 11:449-452. https://doi.org/10.1016/j. parkreldis.2005.05.008

5. Paci C, Thomas A, Onofrj M (2001) Amantadine for dyskinesia in patients affected by severe Parkinson's disease. Neurol Sci 22:7576. https://doi.org/10.1007/s100720170054

6. Jankovic J (2006) An update on the treatment of Parkinson's disease. Mt Sinai J Med 73:682-689

7. Chase TN, Bibbiani F, Oh JD (2003) Striatal glutamatergic mechanisms and extrapyramidal movement disorders. Neurotox Res 5: 139-146

8. Chen X, Wang Y, Wu H, Cheng C, le W (2020) Research advances on L-DOPA-induced dyskinesia: from animal models to human disease. Neurol Sci 41:2055-2065. https://doi.org/10.1007/ s10072-020-04333-5

9. Petrov D, Pedros I, de Lemos ML, Pallàs M, Canudas AM, Lazarowski A, Beas-Zarate C, Auladell C, Folch J, Camins A (2014) Mavoglurant as a treatment for Parkinson's disease. Expert Opin Investig Drugs 23:1165-1179. https://doi.org/10. 1517/13543784.2014.931370

10. Berg D, Godau J, Trenkwalder C, Eggert K, Csoti II, Storch A, Huber H, Morelli-Canelo M, Stamelou M, Ries V, Wolz M, Schneider C, di Paolo T, Gasparini F, Hariry S, Vandemeulebroecke M, Abi-Saab W, Cooke K, Johns D, Gomez-
Mancilla B (2011) AFQ056 treatment of levodopa-induced dyskinesias: results of 2 randomized controlled trials. Mov Disord 26: 1243-1250. https://doi.org/10.1002/mds.23616

11. Trenkwalder C, Stocchi F, Poewe W, Dronamraju N, Kenney C, Shah A, von Raison F, Graf A (2016) Mavoglurant in Parkinson's patients with 1-Dopa-induced dyskinesias: two randomized phase 2 studies. Mov Disord 31:1054-1058. https://doi.org/10.1002/mds. 26585

12. Moher D, Liberati A, Tetzlaff J, Altman DG (2009) Preferred reporting items for systematic reviews and meta-analyses: the PRISMA statement. PLoS Med 6:e1000097. https://doi.org/10. 1371/journal.pmed.1000097

13. Higgins JP, Green S (2008) Cochrane handbook for systematic reviews of interventions: Cochrane book series

14. Owens DK, Lohr KN, Atkins D, et al Methods guide for comparative effectiveness reviews. Rockville, MD Agency Healthc Res Qual

15. Kumar R, Hauser RA, Mostillo J, Dronamraju N, Graf A, Merschhemke M, Kenney C (2016) Mavoglurant (AFQ056) in combination with increased levodopa dosages in Parkinson's disease patients. Int J Neurosci 126:20-24. https://doi.org/10.3109/ 00207454.2013.841685

16. Stocchi F, Rascol O, Destee A, Hattori N, Hauser RA, Lang AE, Poewe W, Stacy M, Tolosa E, Gao H, Nagel J, Merschhemke M, Graf A, Kenney C, Trenkwalder C (2013) AFQ056 in Parkinson patients with levodopa-induced dyskinesia: 13-week, randomized, dose-finding study. Mov Disord 28:1838-1846. https://doi.org/10. $1002 /$ mds.25561

Publisher's note Springer Nature remains neutral with regard to jurisdictional claims in published maps and institutional affiliations. 